\begin{tabular}{|c|l|}
\hline Title & Brown-Halmos type theorems of weighted Toeplitz operators \\
\hline Author(s) & Nakazi, T. \\
\hline Citation & Hokkaido University Preprint Series in Mathematics, 373, 1-14 \\
\hline Issue Date & 1997-4-1 \\
\hline DOI & 10.14943/83519 \\
\hline Doc URL & http://hdl.handle.net/2115/69123 \\
\hline Type & bulletin (article) \\
\hline File Information & pre373.pdf \\
\hline
\end{tabular}

Instructions for use 


\title{
Brown-Halmos Type Theorems \\ Of Weighted Toeplitz Operators
}

\author{
Takahiko Nakazi
}

Series \#373. April 1997 


\section{HOKKAIDO UNIVERSITY PREPRINT SERIES IN MATHEMATICS}

\#349 R. Agemi and K. Yokoyama, The null condition and global existence of solutions to systems of wave equations with different speeds, 42 pages. 1996

\#350 F. Hiroshima, Weak coupling limit with a removal of an ultraviolet cut-off for a Hamiltonian of particles interacting with a massive scalar field, 21 pages. 1996.

\#351 T. Nakazi and Y. Watatani, Invariant subspace theorems for subdiagonal algebras, 22 pages. 1996.

\#352 Y. Nishiura and H. Suzuki, Nonexistence of stable turing patterns with smooth limiting interfacial configurations in higher dimensional spaces, 21 pages. 1996.

\#353 Y.-G. Chen, Y. Giga and K. Sato, On instant extinction for very fast diffusion equations, 9 pages. 1996.

\#354 A. Gyoja and H. Yamashita, Associated variety, Kostant-Sekiguchi correspondence, and locally free $U(\mathrm{n})$-action on Harish-Chandra modules, 25 pages. 1996.

\#355 G. Ishikawa, Topology of plane trigonometric curves and the strangeness of plane curves derived from real pseudo-line arrangements, 18 pages. 1996.

\#356 N.H. Bingham and A. Inoue, The Drasin-Shea-Jordan theorem for Hankel transforms of arbitrarily large order, 13 pages. 1996.

\#357 S. Izumiya, Singularities of solutions for first order partial differential equations, 20 pages. 1996.

\#358 N. Hayashi, P.I. Naumkin and T. Ozawa, Scattering theory for the Hartree equation, 14 pages. 1996.

\#359 I. Tsuda and K. Tadaki, A logic-based dynamical theory for a genesis of biological threshold, 49 pages. 1996.

\#360 I. Tsuda and A. Yamaguchi, Singular-continuous nowhere-differentiable attractors in neural systems, 40 pages. 1996.

\#361 M. Nakamura and T. Ozawa, Low energy scattering for nonlinear Schrödinger equations in fractional order Sobolev spaces, 17 pages. 1996.

\#362 I. Nakamura, Hilbert schemes and simple singularities $E_{6}, E_{7}$ and $E_{8}, 21$ pages. 1996.

\#363 T. Mikami, Equivalent conditions on the central limit theorem for a sequence of probability measures on $R, 7$ pages. 1996.

\#364 S. Izumiya and T. Sano, Generic affine differential geometry of space curves, 23 pages. 1996.

\#365 T. Tsukada, Stability of reticular optical caustics, 12 pages. 1996.

\#366 A. Arai and M. Hirokawa, On the existence and uniqueness of ground states of a generalized spin-boson model, 40 pages. 1996.

\#367 A. Arai, A class of representations of the *-algebra of the canonical commutation relations over a Hilbert space and instability of embedded eigenvalues in quantum field models, 12 pages. 1996.

\#368 K. Ito, BV-solutions of a hyperbolic-elliptic system for a radiating gas, 33 pages. 1997.

\#369 M. Nakamura and T. Ozawa, Nonlinear Schrödinger equations in the Sobolev space of critical order, 20 pages. 1997.

\#370 N.H. Bingham and A. Inoue, An Abel-Tauber theorem for Hankel transforms, 8 pages. 1997.

\#371 T. Nakazi and H. Sawada, The commutator ideal in Toeplitz algebras for uniform algebras and the analytic structure, 9 pages. 1997.

\#372 M.-H. Giga and Y. Giga, Stability for evolving graphs by nonlocal weighted curvature, 70 pages. 1997. 
Brown-Halmos Type Theorems Of Weighted Toeplitz Operators

By

Takahiko Nakazi *

Department of Mathematics

Faculty of Science

Hokkaido University

Sapporo 060, Japan

* This research was partially supported by Grant-in-Aid for Scientific Research, Ministry of Education.

1991 Mathematics Subject Classification. Primary 47 B 35.

Key words and phrases : Toeplitz operator, singular integral operator, weighted Hardy space, spectrum. 
Abstract. The spectra of the Toeplitz operators on the weighted Hardy space $H^{2}(W d \theta / 2 \pi)$ and the Hardy space $H^{p}(d \theta / 2 \pi)$, and the singular integral operators on the Lebesgue space $L^{2}(d \theta / 2 \pi)$ are studied. For example, the theorems of Brown-Halmos type and Hartman-Wintner type are studied. 


\section{$\S 1$. Introduction}

Let $m$ be the normalized Lebesgue measure on the unit circle $T$ and let $W$ be a non-negative integrable function on $T$ which does not vanish identically. Suppose $1 \leq p \leq \infty$. Let $L^{p}(W)=L^{p}(W d m)$ and $L^{p}(W)=L^{p}$ when $W \equiv 1$. Let $H^{p}(W)$ denote the closure in $L^{p}(W)$ of the set $\mathcal{P}$ of all analytic polynomials when $p \neq \infty$. We will write $H^{p}(W)=H^{p}$ when $W \equiv 1$, and then this is a usual Hardy space. $H^{\infty}$ denotes the weak * closure of $\mathcal{P}$ in $L^{\infty} . P$ denotes the projection from the set $\mathcal{C}$ of all trigonometric polynomials to $\mathcal{P}$. For $1<p<\infty, P$ can be extended to a bounded map of $L^{p}(W)$ onto $H^{p}(W)$ if and only if $W$ satisfies the condition

$$
\left(A_{p}\right) \sup _{I}\left(\frac{1}{|I|} \int_{I} W d m\right)\left(\frac{1}{|I|} \int_{I} W^{-\frac{1}{p-1}} d m\right)^{p-1}<\infty
$$

where the supremum is over all intervals $I$ of $T$. This is the well known theorem of Hunt, Muckenhoupt and Wheeden [7], which is a generalization of the theorem of Helson and Szegö [6].

In this paper, we assume that the weight $W$ satisfies the condition $\left(A_{p}\right)$. For $\phi$ in $L^{\infty}$, the Toeplitz operator $T_{\phi}^{W}$ is defined as a bounded map on $H^{p}(W)$ by

$$
T_{\phi}^{W} f=P(\phi f)
$$

For $\alpha$ and $\beta$ in $L^{\infty}$, the singular integral operator $S_{\alpha \beta}^{W}$ is defined as a bounded map on $L^{p}(W)$ by

$$
S_{\alpha \beta}^{W} f=\alpha P f+\beta(I-P) f
$$

where $I$ is an identity operator. If $W \equiv 1$, we will write $T_{\phi}^{W}=T_{\phi}$ and $S_{\alpha \beta}^{W}=S_{\alpha \beta}$. Almost all results in this paper will be essentially shown using the following theorems. They are called the theorems of Widom, Devinatz and Rochberg (cf. [1], [10] and [9]).

Theorem A. Suppose $1<p<\infty$ and $W=|h|^{p}$ satisfies the condition $\left(A_{p}\right)$, where $h$ is an outer function in $H^{p}$. Then the following conditions on $\phi$ and $W$ are equivalent.

(1) $T_{\phi}^{W}$ is an invertible operator on $H^{p}(W)$.

(2) $\phi=k\left(\bar{h}_{0} / h_{0}\right)(h / \bar{h})$, where $k$ is an invertible function in $H^{\infty}$ and $h_{0}$ is an outer function in $H^{p}$ with $\left|h_{0}\right|^{p}$ satisfying the condition $\left(A_{p}\right)$.

(3) $\phi=\gamma \exp (U-i \tilde{V})$, where $\gamma$ is constant with $|\gamma|=1, U$ is a bounded real function, $V$ is a real function in $L^{1}$ and $W \exp \left(\frac{p}{2} V\right)$ satisfies $\left(A_{p}\right)$.

Theorem B. Suppose $1<p<\infty$ and $W=|h|^{p}$ satisfies the condition $\left(A_{p}\right)$, where $h$ is an outer function in $H^{p} . S_{\alpha \beta}^{W}$ is invertible on $L^{p}(W)$ if and only if both $\alpha$ and 
$\beta$ are invertible in $L^{\infty}$ and $\alpha / \beta=\gamma \exp (U-i \tilde{V})$, where $\gamma$ is constant with $|\gamma|=1, U$ is a bounded real function, $V$ is a real function in $L^{1}$ and $W \exp \left(\frac{p}{2} V\right)$ satisfies $\left(A_{p}\right)$.

Theorem C. Suppose $T_{\phi}$ and $S_{\alpha \beta}$ are on $L^{2}$, where $\phi, \alpha$ and $\beta$ are invertible functions in $L^{\infty}$.

(1) $T_{\phi}$ is invertible if and only if $\phi$ has the form : $\phi=|\phi| e^{i t}$ where $t$ is a real function in $L^{1}$ such that

$$
\|t\|^{\prime}=\inf \left\{\|t-\tilde{s}-a\|_{\infty} ; s \in L_{R}^{\infty} \text { and } a \in R\right\}<\pi / 2
$$

(2) $S_{\alpha \beta}$ is invertible if and only if $\alpha / \beta$ has the form : $\alpha / \beta=|\alpha / \beta| e^{i t}$ where $t$ is the same to that of (1). Hence $S_{\alpha \beta}$ is invertible if and only if $T_{\alpha / \beta}$ is invertible. and $S_{\alpha \beta}^{W}$

In this paper, we are interested in $\sigma\left(T_{\phi}^{W}\right)$ and $\sigma\left(S_{\alpha \beta}^{W}\right)$, that is , the spectra of $T_{\phi}^{W}$

For $\alpha=\alpha_{1}+i \alpha_{2} \in \mathbf{C}$ and $\beta=\beta_{1}+i \beta_{2} \in \mathbf{C}$, put $\langle\alpha, \beta\rangle=\alpha_{1} \beta_{1}+\alpha_{2} \beta_{2}$ and $\theta(\alpha, \beta)=\arccos (\langle\alpha, \beta\rangle /|\alpha \| \beta|)$ for $\alpha \neq 0$ and $\beta \neq 0$. Set

$$
\ell_{\alpha}^{+}=\{z \in \mathbf{C} ;\langle z, \alpha\rangle \geq 1\} \text { and } \ell_{\alpha}^{-}=\{z \in \mathbf{C} ;\langle z, \alpha\rangle \leq 1\}
$$

and $\mathcal{E}_{\alpha \beta}^{i j}$ denotes $\ell_{\alpha}^{i} \cap \ell_{\beta}^{j}$ where $i=+$ or - and $j=+$ or - . For each pair $(\alpha, \beta)$,

$$
\mathrm{C}=\mathcal{E}_{\alpha \beta}^{++} \cup \mathcal{E}_{\alpha \beta}^{+-} \cup \mathcal{E}_{\alpha \beta}^{-+} \cup \mathcal{E}_{\alpha \beta}^{--}
$$

and if $\ell=-i$ and $m=-j$, then

$$
\overline{\left(\mathcal{E}^{\ell m}\right)^{c}}=\overline{\mathrm{C} \backslash \mathcal{E}^{\ell m}} \supset \mathcal{E}_{\alpha \beta}^{i j} .
$$

For any bounded subset $E$ in $\mathbf{C}$, there exists a pair $(\alpha, \beta)$ such that $\mathcal{E}_{\alpha \beta}^{i j} \supseteq E$ for some $(i, j)$. In fact, there are a lot of such pairs $(\alpha, \beta)$. Now we can define a set which contains $E$ and is important in this paper. When $|\theta(\alpha, \beta)|=\pi-2 t$ and $0 \leq t \leq \pi / 2$, put

$$
h^{t}(E)=\cap\left\{\overline{\left(\mathcal{E}_{\alpha \beta}^{\ell m}\right)^{c}} ; \mathcal{E}_{\alpha \beta}^{i j} \supseteq E \text { and } \ell=-i, m=-j\right\}
$$

for a subset $E$ in C. If $t<s$, then $h^{t}(E) \subseteq h^{s}(E)$. If $t=0$, then $h^{0}(E)$ is the closed convex hull of $E$. For example, if $E=[a, b]$ then

$$
h^{t}(E)=\triangle(c, r) \cap \triangle(\bar{c}, r)
$$

$c=\frac{a+b}{2}-i \frac{a-b}{2} \cot 2 t$ and $r=-\frac{a-b}{2 \sin 2 t}$ where $\Delta(c, r)$ denotes the circle of center $c$ and radius $r$. If $E=\triangle(0,1)$, then $h^{t}(E)=\triangle(0,1 / \cos t)$. When $T_{\phi}$ is a Toeplitz operator 
on $H^{2}$, Brown and Halmos (cf. [2, Corollary 7.19]) showed that $\sigma\left(T_{\phi}\right) \subseteq h^{0}(\mathcal{R}(\phi))$ where $\mathcal{R}(\phi)$ is the essential range of $\phi$. In this paper we show this type results for Toeplitz operators on $H^{2}(W)$ and $H^{p}$ and for singular integral operators on $L^{2}$. When $\phi$ is a real function and $T_{\phi}$ is a Toeplitz operator on $H^{2}$, Hartman and Wintner (cf. [2, Theorem 7.20]) showed that $\sigma\left(T_{\phi}\right)=h^{0}(\mathcal{R}(\phi))$. In this paper, for real symbols we try to describe the spectra of Toeplitz operators on $H^{2}(W)$ and $H^{p}$, and singular integral operators on $L^{2}$. When $\phi$ is a continuous function, $\sigma\left(T_{\phi}\right)$ is described using $R(\phi)$ and the winding number of the curve determined by $\phi$ (cf. [2, Corollary 7.28]). In this case it is known that $\sigma\left(T_{\phi}^{W}\right)=\sigma\left(T_{\phi}^{p}\right)=\sigma\left(T_{\phi}\right)$ for arbitrary weight $W$ satisfying the condition $\left(A_{2}\right)$, and for any $p$ with $1<p<\infty, T_{\phi}^{p}$ denotes the Toeplitz operator on $H^{p}$. In this paper, we study symbols $\phi$ such that $\sigma\left(T_{\phi}^{W}\right)=\sigma\left(T_{\phi}\right)$ for arbitrary weight $W$.

Now we collect the notations which will be used in this paper. $R$ is the set of all real numbers and $X_{R}$ denotes the set of the real parts of all elements in $X .[X]^{c \ell}$ denotes the closure of $X . D$ is the open unit disc. $C$ is the set of all continuous functions on $T$. If $v$ is a real function in $L^{1}$, then $\tilde{v}$ denotes the harmonic conjugate function with $v(0)=0$.

§. Toeplitz operators on $H^{2}(W)$.

In this section, we fix arbitrary weight $W$ satisfying the condition $\left(A_{2}\right)$ or equivalently, a Helson-Szegö weight $W$. We call $W$ a Helson-Szegö weight when $W=e^{u+\bar{v}}, u$ and $v$ are functions in $L_{R}^{\infty}$ and $\|v\|_{\infty}<\pi / 2$. For a Helson-Szegö weight $W=e^{u+\tilde{v}}$, put

$$
t_{W}=\|v\|^{\prime}=\inf \left\{\|v-\tilde{s}-a\|_{\infty} ; s \in L_{R}^{\infty}, \quad a \in R\right\} .
$$

When $W \equiv 1,(1)$ of Theorem 1 is a theorem of Brown and Halmos (cf. [2, Corollary 7.19]) and (2) and (3) of Theorem 1 is a theorem of Hartman and Wintner (cf. [2, Theorem 7.20]). When $\phi$ is a piecewise continuous function, $\sigma\left(T_{\phi}^{W}\right)$ is described when $W$ is arbitrary weight [10]. The symbol $\phi$ in Corollary 2 and (3) of Corollary 3 is not necessarily piecewise continuous. It is known that $\sigma\left(T_{\phi}^{W}\right) \neq \sigma\left(T_{\phi}\right)$ for some weight $W$ and some piecewise continuous symbol $\phi(c f$. [4]). In Theorem 2, we determine weight $W$ such that $\sigma\left(T_{\phi}^{W}\right)=\sigma\left(T_{\phi}\right)$ for arbitrary symbol $\phi$ in $L^{\infty}$ and study symbols $\phi$ such that $\sigma\left(T_{\phi}^{W}\right)=\sigma\left(T_{\phi}\right)$ for arbitrary weight $W$. (1) of Corollary 3 is related with a particular (corresponding to $p=2$ ) case of [3, Theorem 6.1 and Corollary 6.2]. For if $\log W \in V M O$ then $\log W=u+\tilde{v}$ for some real functions $u$ and $v$ in $C$. (2) of Corollary 3 shows the known result [10] such that if $\phi$ is continuous, then $\sigma\left(T_{\phi}^{W}\right)=\sigma\left(T_{\phi}\right)$ for arbitrary weight $W$.

Theorem 1. Let $\phi$ be a function in $L^{\infty}$, let $W$ be a Helson-Szegö weight and $t=t_{W}$ 
(1) $\mathcal{R}(\phi) \subseteq \sigma\left(T_{\phi}^{W}\right) \subseteq h^{t}(\mathcal{R}(\phi))$.

(2) if $\phi$ is real valued, $a=e s s \inf \phi$ and $b=e s s \sup \phi$, then

$$
\mathcal{R}(\phi) \subseteq \sigma\left(T_{\phi}^{W}\right) \subseteq \triangle(c, r) \cap \triangle(\bar{c}, r)
$$

where $c=\frac{a+b}{2}-i \frac{a-b}{2} \cos 2 t$ and $r=-\frac{a-b}{2 \sin 2 t}$.

(3) Suppose $W=e^{u+\tilde{v}}$ and $\lambda \in[a, b] \cap \mathcal{R}(\phi)^{c}$ in (2). Then $\frac{\phi-\lambda}{|\phi-\lambda|}=e^{i \ell}$ and $\ell=\pi\left(1-\chi_{E}\right)$ for some measurable set $E$ in $T$ with $0<m(E)<1 . \lambda \in \sigma\left(T_{\phi}^{W}\right)$ if and only if

$$
\left\|\pi \chi_{E}-v\right\|^{\prime} \geq \pi / 2 .
$$

Proof. In (1) and (2), it is well known that $\mathcal{R}(\phi) \subseteq \sigma\left(T_{\phi}^{W}\right)$. Suppose $W=e^{u+\tilde{v}}, u$ and $v$ are functions in $L_{R}^{\infty}$ and $\|v\|_{\infty}<\pi / 2$, and $g^{2}=e^{u+\bar{v}+i(\bar{u}-v)}$. Then $W=|g|^{2}$.

(1) By Theorem A in Introduction, for $\lambda \in \mathrm{C}, T_{\phi-\lambda}^{W}$ is invertible if and only if

$$
T_{\frac{\phi-\lambda}{\phi-\lambda \mid} \frac{g}{g}} \text { is invertible. }
$$

Suppose $|\theta(\alpha, \beta)|=\pi-2 t$ and $\mathcal{R}(\phi) \subseteq \mathcal{E}_{\alpha \beta}^{i j}$. If $\lambda \in\left(\mathcal{E}_{\alpha \beta}^{\ell m}\right)^{0}$ with $\ell=-i, m=-j$, then $T_{\phi}^{W}$ is invertible. In fact, then $(\phi-\lambda) /|\phi-\lambda|=e^{i s_{\lambda}}$ where $0 \leq s_{\lambda} \leq \pi-2 t-2 \varepsilon$ a.e. or $-\pi+2 t+2 \varepsilon \leq s_{\lambda} \leq 0$ a.e. for some $\varepsilon>0$. Hence $\left|s_{\lambda}-\frac{\pi}{2}+t+\varepsilon\right| \leq \frac{\pi}{2}-t-\varepsilon$ a.e. or $\left|s_{\lambda}+\frac{\pi}{2}-t-\varepsilon\right| \leq \frac{\pi}{2}-t-\varepsilon$ a.e.. Hence

$$
\frac{\phi-\lambda}{|\phi-\lambda|} \frac{\bar{g}}{g}=e^{i\left(s_{\lambda}+v-\tilde{u}\right)}
$$

and

$$
\left\|s_{\lambda}+v-\tilde{u}\right\|^{\prime} \leq \frac{\pi}{2}-\varepsilon .
$$

Thus $T_{\frac{\phi-\lambda}{[\phi-\lambda \mid}} \frac{g}{g}$ is invertible by Theorem $\mathrm{C}$ and hence $T_{\phi-\lambda}^{W}$ is invertible. If $\lambda \notin h^{t}(\mathcal{R}(\phi))$, then by definition $\lambda \in \cup\left\{\left(\mathcal{E}_{\alpha \beta}^{\ell m}\right)^{0} ; \mathcal{E}_{\alpha \beta}^{i j} \supseteq \mathcal{R}(\phi)\right.$ and $\left.\ell=-i, m=-j\right\}$ and $|\theta(\alpha, \beta)|=$ $\pi-2 t$. By what was just proved, $\lambda \notin \sigma\left(T_{\phi}^{W}\right)$. (2) By (1), $\sigma\left(T_{\phi}^{W}\right) \subseteq h^{t}(\mathcal{R}(\phi)) \subseteq h^{t}([a, b])$ for $t=t_{W}$. It is elementary to see that $h^{t}([a, b]) \subseteq \triangle(c, r) \cap \triangle(\bar{c}, r)$. (3) The first part is clear. The second statement is a result of Theorems $A$ and $C$.

Corollary 1. Suppose $\phi=a \chi_{E}+b \chi_{E^{c}}$ where $a$ and $b$ are real numbers, $a \neq b$ and $0<m(E)<1$. Let $W=e^{u+\bar{v}}$, then $\sigma\left(T_{\phi}^{W}\right) \supseteq[a, b]$ if and only if $\left\|\pi \chi_{E}-v\right\|^{\prime} \geq \pi / 2$.

Corollary 2. Let $E$ be a measurable set with $0<m(E)<1$. Suppose $W$ and $\phi$ satisfy the following (i) and (ii) : 
(i) $W=e^{u+\tilde{v}}$ where $u \in L_{R}^{\infty}, \tilde{v}=d\left(\chi_{E}-\chi_{E^{c}}\right)+q, q \in C_{R}$ and $d$ is a constant with $0<d<\pi / 2$.

(ii) $\phi=a \chi_{E}+b \chi_{E^{c}}$ where $a$ and $b$ are real numbers.

Then $t_{W}=d$,

$$
\sigma\left(T_{\phi}^{W}\right)=\left\{\lambda \in \mathbf{C} ; \arg \frac{a-\lambda}{b-\lambda}=\pi-2 d\right\} .
$$

and

$$
h^{d}(\mathcal{R}(\phi))=\left\{\lambda \in \mathrm{C} ; \arg \frac{a-\lambda}{b-\lambda}=\pi-2 d \text { or }-\pi+2 d\right\} .
$$

Proof. Put $v_{0}=\frac{\pi}{2}\left(\chi_{E}-\chi_{E^{c}}\right)$, then $h^{2}=e^{\tilde{v}_{0}-i v_{0}}$ and $|h|^{2} / h^{2}=e^{i v_{0}}=i\left(\chi_{E}-\chi_{E^{c}}\right)$. If $\left\|\chi_{E}-\chi_{E^{c}}\right\|^{\prime}<1$, then $|h|^{2}=e^{\bar{v}_{0}}$ is a Helson-Szegö weight and so $\left\||h|^{2} / h^{2}+z H^{\infty}\right\|<$ 1 (see [3, Chapter IV, Theorem 3.1]). On the other hand, $\left\||h|^{2} / h^{2}+z H^{\infty}\right\|=\| i\left(\chi_{E}-\right.$ $\left.\chi_{E^{c}}\right)+z H^{\infty} \|=1$. This contradiction shows that $\left\|\chi_{E}-\chi_{E^{c}}\right\|^{\prime}=1$. Thus

$$
\begin{aligned}
t_{W} & =\inf \left\{\left\|d\left(\chi_{E}-\chi_{E^{c}}\right)-\tilde{s}-a\right\|_{\infty} ; s \in L_{R}^{\infty}, a \in R\right\} \\
& =d \inf \left\{\left\|\chi_{E}-\chi_{E^{c}}-\tilde{s}-a\right\|_{\infty} ; s \in L_{R}^{\infty}, a \in R\right\} \\
& =d .
\end{aligned}
$$

Put $g^{2}=e^{u+\tilde{u}+i(\tilde{u}-v)}$, then $\bar{g} / g=e^{i(\tilde{u}-v)}=\exp i\left\{\tilde{u}-d\left(\chi_{E}-\chi_{E^{c}}\right)-q\right\}$. If $\lambda \neq a$ and $\lambda \neq b$, then

$$
\begin{aligned}
\frac{\phi-\lambda}{|\phi-\lambda|} & =\frac{a-\lambda}{|a-\lambda|} \chi_{E}+\frac{b-\lambda}{|b-\lambda|} \chi_{E^{c}} \\
& =\exp i\left\{a(\lambda) \chi_{E}+b(\lambda) \chi_{E^{c}}\right\}
\end{aligned}
$$

where $a(\lambda)=\arg (a-\lambda)$ and $b(\lambda)=\arg (b-\lambda)$. Thus $(\phi-\lambda) \bar{g} /|\phi-\lambda| g=\exp i\left\{a(\lambda) \chi_{E}+\right.$ $\left.b(\lambda) \chi_{E^{c}}+\tilde{u}-d\left(\chi_{E}-\chi_{E^{c}}\right)-q\right\}$. Since $q \in C_{R}$, by the first part of the proof,

$$
\begin{aligned}
\inf & \left\{\left\|a(\lambda) \chi_{E}+b(\lambda) \chi_{E^{c}}-d\left(\chi_{E}-\chi_{E^{c}}\right)+\tilde{u}-q-\tilde{s}-a\right\|_{\infty} ; s \in L_{R}^{\infty}, a \in R\right\} \\
= & \left|\frac{a(\lambda)-b(\lambda)}{2}-d\right| \inf \left\{\left\|\chi_{E}-\chi_{E^{c}}-\tilde{s}-a\right\|_{\infty} ; s \in L_{R}^{\infty}, a \in R\right\} \\
= & \left|\frac{a(\lambda)-b(\lambda)}{2}-d\right|=\frac{1}{2}\left|\arg \frac{a-\lambda}{b-\lambda}-2 d\right| .
\end{aligned}
$$

Thus, by (1) of Theorem $\mathrm{C} \lambda \notin \sigma\left(T_{\phi}^{W}\right)$ if and only if $\left|\arg \frac{a-\lambda}{b-\lambda}-2 d\right| \neq \pi$. If $\arg \frac{a-\lambda}{b-\lambda}>$ 0 , then $\left|\arg \frac{a-\lambda}{b-\lambda}-2 d\right| \neq \pi$ because $d>0$, and hence $\sigma\left(T_{\phi}^{W}\right)=\left\{\lambda \notin \mathrm{C} ; \arg \frac{a-\lambda}{b-\lambda}=\right.$ $\pi-2 d\}$. The description of $h^{d}(\mathcal{R}(\phi))$ is a result of (2) of Theorem 1 .

Theorem 2. Let $\phi$ be a function in $L^{\infty}$ and let $W$ be a Helson-Szegö weight. 
(1) $t_{W}=0$ if and only if $\sigma\left(T_{\phi}^{W}\right)=\sigma\left(T_{\phi}\right)$ for arbitrary symbol $\phi$ in $L^{\infty}$.

(2) $\sigma\left(T_{\phi}\right) \supseteq \sigma\left(T_{\phi}^{W}\right)$ for arbitrary Helson-Szegö weight $W$ if and only if for any $\lambda \notin \sigma\left(T_{\phi}\right), \frac{\phi-\lambda}{|\phi-\lambda|}=e^{i \ell}$ and $\|\ell\|^{\prime}=0$.

Proof. (1) Suppose $W=e^{u+\tilde{v}}, t_{W}=0$ and $g^{2}=e^{u+\bar{v}+i(\bar{u}-v)}$. If $\lambda \notin \sigma\left(T_{\phi}\right)$, then by Theorem $\mathrm{C} \frac{\phi-\lambda}{|\phi-\lambda|}=e^{i \ell}$ and $\|\ell\|^{\prime}<\pi / 2$. Hence

$$
\frac{\phi-\lambda}{|\phi-\lambda|} \frac{\bar{g}}{g}=\exp i(\ell+\tilde{u}-v)
$$

and since $t_{W}=0$,

$$
\begin{aligned}
& \inf \left\{\|\ell+\tilde{u}-v-\tilde{s}-a\|_{\infty} ; s \in L_{R}^{\infty} \text { and } a \in R\right\} \\
& =\inf \left\{\|\ell-\tilde{s}-a\|_{\infty} ; s \in L_{R}^{\infty} \text { and } a \in R\right\} \\
& <\frac{\pi}{2}
\end{aligned}
$$

Thus $\lambda \notin \sigma\left(T_{\phi}^{W}\right)$ by Theorems $A$ and $C$. Similarly we can show that if $\lambda \notin \sigma\left(T_{\phi}^{W}\right)$ then $\lambda \in \sigma\left(T_{\phi}\right)$. Suppose $\sigma\left(T_{\phi}^{W}\right)=\sigma\left(T_{\phi}\right)$ for arbitrary symbol $\phi$ in $\mathrm{L}^{\infty}$. If $t=t_{W}$ is nonzero and $W=e^{u+\tilde{v}}$ is a Helson-Szegö weight, then $T_{\phi}$ is invertible where $\phi=e^{-i k v}$ and $k=\pi / 2 t-1$. For $\inf \left\{\|k v-\tilde{s}-a\|_{\infty} ; s \in L_{R}^{\infty}\right.$ and $\left.a \in R\right\}=k t=\pi / 2-1$. On the other hand, $T_{\phi}^{W}$ is not invertible. For

$$
\frac{\phi}{|\phi|} \frac{\bar{g}}{g}=\exp i\{\tilde{u}-(k+1) v\}
$$

and

$$
\inf \left\{\|\tilde{u}-(k+1) v-\tilde{s}-a\|_{\infty} ; s \in L_{R}^{\infty} \text { and } a \in R\right\}=(k+1) t=\frac{\pi}{2}
$$

where $g^{2}=e^{u+\tilde{v}+i(\tilde{u}-v)}$

(2) Suppose for any $\lambda \notin \sigma\left(T_{\phi}\right), \frac{\phi-\lambda}{|\phi-\lambda|}=e^{i \ell}$ and $\inf \left\{\|\ell-\tilde{s}-a\|_{\infty} ; s \in L_{R}^{\infty}\right.$ and $a \in R\}=0$. We will show that $\sigma\left(T_{\phi}\right) \supseteq \sigma\left(T_{\phi}^{W}\right)$ for arbitrary Helson-Szegö weight $W$. If $\lambda \notin \sigma\left(T_{\phi}\right), W=e^{u+\bar{v}}$ is a Helson-Szegö weight and $g^{2}=e^{u+\tilde{v}+i(\tilde{u}-v)}$, then

$$
\frac{\phi-\lambda}{|\phi-\lambda|} \frac{\bar{g}}{g}=e^{i(\ell+\tilde{u}-v)}
$$

and $\inf \left\{\|\ell+\tilde{u}-v-\tilde{s}-a\|_{\infty} ; s \in L_{R}^{\infty}, a \in R\right\}<\pi / 2$ by the hypothesis. This implies that $\sigma\left(T_{\phi}^{W}\right) \not \supset \lambda$. Conversely suppose that $\sigma\left(T_{\phi}\right) \supseteq \sigma\left(T_{\phi}^{W}\right)$ for arbitrary Helson-Szegö weight $W$. If $\lambda \notin \sigma\left(T_{\phi}\right)$, then $\frac{\phi-\lambda}{|\phi-\lambda|}=e^{i \ell}$ and $b=\inf \left\{\|\ell-\tilde{s}-a\|_{\infty} ; s \in L_{R}^{\infty}, a \in R\right\}<\pi / 2$. If $b \neq 0$, put $W=e^{k \bar{\ell}}$ and $g^{2}=e^{k \bar{\ell}-i k \ell}$ where $k=\frac{\pi}{2 b}-1$, then $W$ is a Helson-Szegö weight. However $T_{\phi}^{W}$ is not invertible and so $\lambda \in \sigma\left(T_{\phi}^{W}\right)$. This contraciction implies that $b=0$. 
Corollary 3. Let $\phi$ be a function in $L^{\infty}$.

(1) If $W=e^{u+\bar{v}}, u$ and $v$ are real functions in $L^{\infty}$ and $C$ respectively, then $\sigma\left(T_{\phi}^{W}\right)=\sigma\left(T_{\phi}\right)$ for arbitrary symbol $\phi$ in $L^{\infty}$. weight $W$.

(2) If $\phi$ is a function in $C$ or $H^{\infty}$, then $\sigma\left(T_{\phi}^{W}\right)=\sigma\left(T_{\phi}\right)$ for arbitrary Helson-Szego"

(3) If $\phi=a \chi_{E}+b \chi_{E^{c}}, 0<m(E)<1$ and $a, b \in \mathrm{C}$ with $a \neq b$, then there exists a Helson-Szegö weight $W$ such that $\sigma\left(T_{\phi}^{W}\right) \subset_{\mp} \sigma\left(T_{\phi}\right)$.

Proof. Since $t_{W}=0$ because $v \in C_{R}$, (1) of Theorem 2 implies (1). Suppose $\phi$ is a function in $C$ and $\lambda \notin \sigma\left(T_{\phi}^{W^{\prime}}\right)$ for a Helson-Szegö weight $W^{\prime}=e^{u+\bar{v}}$. Since $\mathcal{R}(\phi) \subseteq \sigma\left(T_{\phi}^{W^{\prime}}\right)$,

$$
\frac{\phi-\lambda}{|\phi-\lambda|} \frac{\bar{g}}{g}=z^{m} e^{i \ell} e^{i(\tilde{u}-v)}
$$

where $m$ is an integer, $\ell \in C_{R}$ and $g^{2}=e^{u+\bar{v}+i(\bar{u}-v)}$. By Therems $A$ and $C$, we can show $m=0$. As $W^{\prime} \equiv 1,(2)$ of Therem 2 implies that $\sigma\left(T_{\phi}\right) \supseteq \sigma\left(T_{\phi}^{W}\right)$ for arbitrary HelsonSzegö weight $W$. The converse is trivial. Suppose $\phi$ is a function in $H^{\infty}$ and $\lambda \notin \sigma\left(T_{\phi}^{W^{\prime}}\right)$ for a Helson Szegö weight $W^{\prime}=e^{u+\bar{v}}$. Since $\mathcal{R}(\phi) \subseteq \sigma\left(T_{\phi}^{W^{\prime}}\right), \phi-\lambda$ is invertible in $L^{\infty}$ and so $\phi-\lambda=q h$ where $q$ is inner and $h$ is invertible in $H^{\infty}$. Since $h=e^{\ell+i \bar{\ell}}$ and $\ell=\log |h| \in L^{\infty}$,

$$
\frac{\phi-\lambda}{|\phi-\lambda|} \frac{\bar{g}}{g}=q e^{i \tilde{\ell}} e^{i(\tilde{u}-v)}
$$

where $g^{2}=e^{u+\tilde{v}+i(\tilde{u}-v)}$. By Theorems $A$ and $C$, we can show that $q$ is constant. As in case $\phi \in C$, we can show $\sigma\left(T_{\phi}^{W}\right)=\sigma\left(T_{\phi}\right)$ for arbitrary Helson-Szegö weight $W$. This completes the proof of (2). Suppose $\phi=a \chi_{E}+b \chi_{E^{c}}, 0<m(E)<1$ and $a, b \in \mathbf{C}$ with $a \neq b$. To prove (3), without loss of generality, we may assume that $a$ and $b$ are real numbers. By a theorem of Hartman and Wintner (cf.[2, Theorem 7.20]), $\sigma\left(T_{\phi}\right)=[a, b]$. If $\lambda \notin[a, b]$,

$$
\frac{\phi-\lambda}{|\phi-\lambda|}=\exp i\left\{a(\lambda) \chi_{E}+b(\lambda) \chi_{E^{c}}\right\}
$$

where $a(\lambda)=\arg (a-\lambda)$ and $b(\lambda)=\arg (b-\lambda)$. By the proof of Corollary 1 ,

$$
\inf \left\{\left\|a(\lambda) \chi_{E}+b(\lambda) \chi_{E^{c}}-\tilde{s}-a\right\|_{\infty} ; s \in L_{R}^{\infty} \text { and } a \in R\right\}=\frac{1}{2}\left|\arg \frac{a-\lambda}{b-\lambda}\right| \neq 0
$$

and hence by (2) of Theorem 2, there exists a Helson-Szegö weight $W$ such that $\sigma\left(T_{\phi}^{W}\right) \subset_{f} \sigma\left(T_{\phi}\right)$ 


\section{§3. Toeplitz operators on $H^{p}$}

For $1<p<\infty, T_{\phi}^{p}$ denotes a Toeplitz operator on $H^{p}$. We will write $T_{\phi}^{2}=T_{\phi}$. By a theorem of Widom, Devinatz and Rochberg (cf.[8]), we know the invertibility of $T_{\phi}^{p}$ and by a theorem of Widom ( $c f$. [2, Corollary 7.46]), $\sigma\left(T_{\phi}^{p}\right)$ is connected. If $1<q<2<$ $p<\infty$, then $A_{q} \subset A_{2} \subset A_{p}$. It is more difficult to describe $\sigma\left(T_{\phi}^{q}\right)$ than $\sigma\left(T_{\phi}^{p}\right)$. In this paper, we study only $\sigma\left(T_{\phi}^{p}\right)$. When $p=2,(1)$ of Theorem 3 is a theorem of Brown and Halmos and (2) is a theorem of Hartman and Wintner. (3) of Theorem 3 is known in [10] for arbitrary $1<p<\infty$. Our proof is different from it.

Theorem 3. Suppose $p \geq 2$ and $t=(p-2) \pi / 2 p$.

(1) If $\phi$ is a function in $L^{\infty}$, then $\sigma\left(T_{\phi}^{p}\right) \subseteq h^{t}(\mathcal{R}(\phi))$.

(2) If $\phi$ is a real function in $L^{\infty}, a=e s s \inf \phi$ and $b=e s s \sup \phi$, then

$$
[a, b] \subseteq \sigma\left(T_{\phi}^{p}\right) \subseteq \triangle(c, r) \cap \triangle(\bar{c}, r)
$$

where $c=\frac{a+b}{2}-i \frac{a-b}{2} \cot 2 t$ and $r=-\frac{a-b}{2 \sin 2 t}$. In particular, if $p=2$, then $t=0$ and hence $\sigma\left(T_{\phi}^{p}\right)=[a, b]$.

(3) If $\phi$ is a function in $C$, then $\sigma\left(T_{\phi}^{p}\right)=\sigma\left(T_{\phi}\right)$.

Proof. (1) If $\lambda \notin h^{t}(\mathcal{R}(\phi))$, then by definition $\lambda \in \cup\left\{\left(\mathcal{E}_{\alpha \beta}^{\ell m}\right)^{0} ; \mathcal{E}_{\alpha \beta}^{i j} \supseteq \mathcal{R}(\phi)\right.$ and $\ell=-i, m=-j\}$ and $|\theta(\alpha, \beta)|=\pi-2 t$. Hence $(\phi-\lambda) /|\phi-\lambda|=e^{i s_{\lambda}}$ where $0 \leq s_{\lambda} \leq \pi-2 t-2 \varepsilon$ a.e. or $-\pi+2 t+2 \varepsilon \leq s_{\lambda} \leq 0$ a.e. for some $\varepsilon>0$. Put $v_{\lambda}=s_{\lambda}-\frac{\pi}{2}+t+\varepsilon$ or $v_{\lambda}=s_{\lambda}+\frac{\pi}{2}-t-\varepsilon$, then $\left\|v_{\lambda}\right\|_{\infty} \leq \frac{\pi}{2}-t-\varepsilon$. Put $g^{2}=e^{-\tilde{v}_{\lambda}+i v_{\lambda}}$, then $g^{2}$ is an outer function and $|g|^{2}=e^{-\bar{v}_{\lambda}}$. Then $\left\|\frac{p}{2} v_{\lambda}\right\|_{\infty}<\frac{\pi}{2}$ because $\left\|v_{\lambda}\right\|_{\infty}<\frac{\pi}{2}-\frac{(p-2) \pi}{2 p}$. Hence $|g|^{p}$ satisfies $\left(A_{2}\right)$ condition and so $|g|^{p}$ satisfies $\left(A_{p}\right)$ condition by (cf. [3, Lemma $6.8])$ because $p>2$. Since $(\phi-\lambda) /|\phi-\lambda|=\alpha(\bar{g} / g)$ for some constant $\alpha$ with $|\alpha|=1$, Theorem A impties (1).

(2) We may assume that $\phi$ is not constant. By Theorem $\mathrm{A}, \mathcal{R}(\phi) \subseteq \sigma\left(T_{\phi}^{p}\right)$. Suppose $\lambda \in[a, b]$ and $\lambda \notin \mathcal{R}(\phi)$, then $(\phi-\lambda) /|\phi-\lambda|=2 \chi_{E}-1$ for some measurable set $E$ in $T$. If $\lambda \notin \sigma\left(T_{\phi}^{p}\right)$, then by Theorem $A$, there exists an outer function $h_{0}$ in $H^{p}$ such that $2 \chi_{E}-1=\bar{h}_{0} / h_{0}$. This implies that $h_{0}^{2}$ is a real function in $H^{1}$ because $p \geq 2$. It is well known that only one real function in $H^{1}$ is constant. Hence $h_{0}$ is constant and this contradicts that $\phi$ is not constant. Thus $[a, b] \subseteq \sigma\left(T_{\phi}^{p}\right)$. Now (1) implies (2).

(3) If $\lambda \notin \mathcal{R}(\phi)$, then $(\phi-\lambda) /|\phi-\lambda|$ is a continuous function and hence

$$
\frac{\phi-\lambda}{|\phi-\lambda|}=z^{\ell} e^{i v}
$$

where $\ell$ is an integer and $v$ is a real function in $C$. Put $g^{2}=e^{-\bar{v}+i v}$, then $|g|^{2}=e^{-\bar{v}}$. Since $v$ is continuous, for any $\varepsilon>0, \tilde{v}=s+\tilde{t}$ where both $s$ and $t$ are in $C$ and $\|t\|_{\infty}<\varepsilon$. 
Suppose $\ell=0$. If $\varepsilon<\pi / p$, then $|g|^{p}=\left|g^{2}\right|^{\frac{p}{2}}=\exp \left(-\frac{p}{2} \tilde{v}\right)=\exp \left(-\frac{p}{2} s-\frac{p}{2} \tilde{t}\right)$ and $\left\|\frac{p}{2} t\right\|_{\infty}<\frac{\pi}{2}$. Hence $|g|^{p}$ satisfies $\left(A_{2}\right)$ condition and so $\left(A_{p}\right)$. By Theorem A, $T_{\phi-\lambda}^{p}$ is invertible and so $\lambda \notin \sigma\left(T_{\phi}^{p}\right)$. Suppose $\ell \neq 0$. If $T_{\phi-\lambda}^{p}$ is invertible, then by Theorem A

$$
\frac{\phi-\lambda}{|\phi-\lambda|}=z^{\ell} e^{i v}=\frac{|k|}{k} \frac{|h|^{2}}{h^{2}}
$$

where $k$ and $k^{-1}$ are in $H^{\infty}$, and $h$ is an outer function in $H^{p}$ with $|h|^{p}$ satisfying $\left(A_{p}\right)$ condition. Since $z^{\ell}|g|^{2} / g^{2}=\left|k h^{2}\right| / k h^{2}, z^{\ell} f \geq 0$ a.e. where $f=k h^{2} / g^{2}$. If $\ell>0, z^{\ell} f$ is a nonnegative function in $H^{1 / 2}$ and hence it is constant. This contradicts that $z^{\ell}$ is zero on the origin. If $\ell<0, z^{\ell}\left|1+\bar{z}^{\ell}\right| /\left(1+\bar{z}^{\ell}\right)^{2} \geq 0$ and so $\left(1+\bar{z}^{\ell}\right)^{2} f \geq 0$ a.e.. Thus $\left(1+\bar{z}^{\ell}\right)^{2} f$ is a nonnegative function in $H^{1 / 2}$ and so $f=c\left(1+\bar{z}^{\ell}\right)^{2}$ for some constant $c>0$. This contradicts that $f^{-1} \in H^{1 / 2}$.

$\S 4$. Singular integral operators on $L^{2}$

By Theorems A, B and $\mathrm{C}$, we can expect that $\sigma\left(S_{\alpha \beta}\right)$ is strongly related with $\sigma\left(T_{\alpha}\right)$ and $\sigma\left(T_{\beta}\right)$. (1) of Theorem 4 is an analogy of a theorem of Brown and Halmos, and (2) of Theorem 4 is an analogy of a theorem of Hartman and Wintner.

Theorem 4. Suppose $\alpha$ and $\beta$ are functions in $L^{\infty}$.

(1) $\mathcal{R}(\alpha) \cup \mathcal{R}(\beta) \subseteq \sigma\left(S_{\alpha \beta}\right) \subseteq h^{t}(\mathcal{R}(\alpha) \cup \mathcal{R}(\beta))$ where $t=\pi / 4$.

(2) If $\alpha$ and $\beta$ are real functions in $L^{\infty}$,

$$
\left\{h(\mathcal{R}(\alpha)) \cap h(\mathcal{R}(\beta))^{c}\right\} \cup\left\{h(\mathcal{R}(\alpha))^{c} \cap h(\mathcal{R}(\beta))\right\} \subseteq \sigma\left(S_{\alpha \beta}\right) \subseteq \triangle(c, r) \cap \triangle(\bar{c}, r)
$$

where $a=\min \{$ ess inf $\alpha$, ess $\inf \beta\}, b=\max \{$ ess $\sup \alpha$, ess $\sup \beta\}, c=\frac{a+b}{2}-i \frac{a-b}{2}$ and $r=-\frac{a-b}{2}$.

(3) If $\beta$ is in $C$,

$$
\sigma\left(T_{\alpha}\right) \cap\left\{\lambda \in \mathbf{C} ; i_{t}(\beta, \lambda)=0\right\} \cup \mathcal{R}(\beta) \subseteq \sigma\left(S_{\alpha \beta}\right) \subseteq \sigma\left(T_{\alpha}\right) \cup \sigma\left(T_{\beta}\right) .
$$

(4) If both $\alpha$ and $\beta$ are in $C$, then $\sigma\left(S_{\alpha \beta}\right)=\left\{\sigma\left(T_{\alpha}\right) \cup \sigma\left(T_{\beta}\right)\right\} \backslash\left\{\lambda \in \mathbf{C} ; i_{t}(\alpha, \lambda)=\right.$ $\left.i_{t}(\beta, \lambda) \neq 0\right\}$.

(5) Suppose both $\alpha$ and $\beta$ are in $C$. If $\beta$ is a real function, then $\sigma\left(S_{\alpha \beta}\right)=\sigma\left(T_{\alpha}\right) \cup$ $h(\mathcal{R}(\beta))$ and hence if both $\alpha$ and $\beta$ are real functions, then $\sigma\left(S_{\alpha \beta}\right)=h(\mathcal{R}(\alpha) \cup h(\mathcal{R}(\beta))$. 
(6) If $\alpha$ and $\bar{\beta}$ are functions in $H^{\infty}$, then $\sigma\left(S_{\alpha \beta}\right)=[\alpha(D)]^{c l} \cup[\overline{\bar{\beta}(D)}]^{c l}$.

(7) If $\alpha$ and $\beta$ are functions in $H^{\infty}$, then $\sigma\left(S_{\alpha \beta}\right)=[\alpha(D)]^{c l} \cup[\beta(D)]^{c l} \backslash\{\lambda \notin$ $\mathcal{R}(\alpha) \cup \mathcal{R}(\beta) ; T_{q_{\lambda} \bar{p}_{\lambda}}$ is invertible $\}$ where $q_{\lambda}$ is the inner part of $\alpha-\lambda$ and $p_{\lambda}$ is the inner part of $\beta-\lambda$.

(8) If $\alpha$ and $\beta$ are inner functions, and $\operatorname{sing} \alpha \neq \operatorname{sing} \beta$, then $\sigma\left(S_{\alpha \beta}\right)=[D]^{c l}$, where $\operatorname{sing} \alpha$ and $\operatorname{sing} \beta$ denote the subsets of $\partial D$ on which $\alpha$ and $\beta$ can not be analytically extended, respectively.

Proof. (1) By Theorem B, it is clear that $\mathcal{R}(\alpha) \cup \mathcal{R}(\beta) \subseteq \sigma\left(S_{\alpha \beta}\right)$. If $\lambda \notin$ $h^{t}(\mathcal{R}(\alpha) \cup \mathcal{R}(\beta))$, then $(\alpha-\lambda) /|\alpha-\lambda|=e^{i s_{\lambda}}$ and $(\beta-\lambda) /|\beta-\lambda|=e^{i t_{\lambda}}$ where $0 \leq s_{\lambda}, t_{\lambda} \leq \frac{\pi}{2}-\varepsilon$ a.e. or $-\frac{\pi}{2}+\varepsilon \leq s_{\lambda}, t_{\lambda} \leq 0$ a.e. fro some $\varepsilon>0$. Therefore

$$
\frac{\alpha-\lambda}{\beta-\lambda}=\exp (U-i \tilde{V})
$$

where $U=\log |\alpha-\lambda|-\log |\beta-\lambda|$ and $\tilde{V}=t_{\lambda}-s_{\lambda}$. Then $U$ is bounded and $\exp V=\exp -\left(t_{\lambda}-s_{\lambda}\right)^{\sim}$ and $\left\|t_{\lambda}-s_{\lambda}\right\|_{\infty} \leq \frac{\pi}{2}-\varepsilon$. By Theorem C, $S_{\alpha-\lambda, \beta-\lambda}$ is invertible.

(2) If $\alpha$ and $\beta$ are real functions and $\lambda \in h(\mathcal{R}(\alpha)) \cap h(\mathcal{R}(\beta))^{c}$, then $\alpha-\lambda$ is a real function which is not nonnegative or nonpositive, and $\beta-\lambda$ is a nonnegative or nonpositive function which is invertible in $L^{\infty} \cdot(\alpha-\lambda) /(\beta-\lambda)$ is a real function in $L^{\infty}$ which is not nonnegative or nonpositive. If $S_{\alpha-\lambda, \beta-\lambda}$ is invertible, then by Theorems B and $\mathrm{C}$ both $\alpha-\lambda$ and $\beta-\lambda$ are invertible in $L^{\infty}$, and

$$
\frac{\alpha-\lambda}{\beta-\lambda}=\left|\frac{\alpha-\lambda}{\beta-\lambda}\right| e^{i t}
$$

where $\inf \left\{\|t-\tilde{s}-a\|_{\infty}: s \in L_{R}^{\infty}\right.$ and $\left.a \in R\right\}<\pi / 2$. Let $g=e^{-\bar{t}+i t}$, then $g$ is a real function in $H^{1}$. Since only one real function in $H^{1}$ is constant, $g$ is constant and so it contradicts that $(\alpha-\lambda)|\beta-\lambda| /(\beta-\lambda)|\alpha-\lambda|$ is nonconstant. This implies that $h(\mathcal{R}(\alpha)) \cap h(\mathcal{R}(\beta))^{c} \subseteq \sigma\left(S_{\alpha \beta}\right)$. The same method shows that $h(\mathcal{R}(\alpha))^{c} \cap h(\mathcal{R}(\beta)) \subseteq$ $\sigma\left(S_{\alpha \beta}\right)$. Since $\mathcal{R}(\alpha) \cup \mathcal{R}(\beta) \subseteq[a, b]$, by $(1) \sigma\left(S_{\alpha \beta}\right) \subseteq h^{t}([a, b])$ where $t=\pi / 4$. This implies (2).

(3) Suppose $\lambda \in \sigma\left(T_{\alpha}\right) \cap\left\{\lambda \in \mathbf{C} ; i_{t}(\beta, \lambda)=0\right\}$. Then $\beta-\lambda=|\beta-\lambda| e^{i v}$ and $v \in C$ because $\beta$ is continuous. If $S_{\alpha-\lambda, \beta-\lambda}$ is invertible, then by Theorem B

$$
\frac{\alpha-\lambda}{\beta-\lambda}=\gamma e^{(U-i \tilde{V})}
$$

where $\gamma$ is constant, $U$ is a bounded real function, $V$ is a real function in $L^{1}$ and $\exp V$ satisfies $\left(A_{2}\right)$ condition. Hence

$$
\alpha-\lambda=\gamma \exp \{U+\log |\beta-\lambda|-i(\tilde{V}-v)\}
$$

,$U+\log |\beta-\lambda|$ is in $L^{\infty}$ and $e^{V-\tilde{v}}$ satisfies $\left(A_{2}\right)$ condition because $v \in C$. By Theorem A, this implies that $\lambda \notin \sigma\left(T_{\alpha}\right)$. This contradiction shows that $\lambda \in \sigma\left(S_{\alpha \beta}\right)$ and hence $\sigma\left(T_{\alpha}\right) \cap\left\{\lambda \in \mathrm{C} ; i_{t}(\beta, \lambda)=0\right\} \cup \mathcal{R}(\beta) \subseteq \sigma\left(S_{\alpha \beta}\right)$. If $\lambda \notin \sigma\left(T_{\alpha}\right) \cup \sigma\left(T_{\beta}\right)$, then by Theorem $\mathrm{C}$ and [2, Corollary 7.28] $\alpha-\lambda=|\alpha-\lambda| e^{i t}$ and $\beta-\lambda=|\beta-\lambda| e^{i \ell}$ where $\inf \left\{\|t-\tilde{s}-a\|_{\infty} ; s \in L_{R}^{\infty}\right.$ and $\left.a \in R\right\}<\pi / 2$ and $\ell \in C$. Therefore 


$$
\frac{\alpha-\lambda}{\beta-\lambda}=\frac{|\alpha-\lambda|}{|\beta-\lambda|} e^{i(t-\ell)}
$$

and hence by Theorem $\mathrm{C} \lambda \notin \sigma\left(S_{\alpha \beta}\right)$.

(4) If $\lambda \notin \mathcal{R}(\alpha) \cup \mathcal{R}(\beta)$ and $i_{t}(\alpha, \lambda) \neq i_{t}(\beta, \lambda)$, then $\alpha-\lambda=|\alpha-\lambda| z^{\ell} e^{i u}$ and $\beta-\lambda=|\beta-\lambda| z^{t} e^{i v}$ where $u$ and $v$ are in $C$, and $\ell$ and $t$ are integers with $\ell \neq t$. Hence

$$
\frac{\alpha-\lambda}{\beta-\lambda}=\frac{|\alpha-\lambda|}{|\beta-\lambda|} z^{\ell-t} e^{i(u-v)}
$$

and $\ell-t \neq 0$. By Theorem $\mathrm{C}$, we can show that $\lambda \notin \sigma\left(S_{\alpha \beta}\right)$. This implies that $\left\{\sigma\left(T_{\alpha}\right) \cup\right.$ $\left.\sigma\left(T_{\beta}\right)\right\} \backslash\left\{\lambda \in \mathbf{C} ; i_{t}(\alpha, \lambda)=i_{t}(\beta, \lambda) \neq 0\right\} \subseteq \sigma\left(S_{\alpha \beta}\right)$. If $\lambda \notin\left\{\sigma\left(T_{\alpha}\right) \cup \sigma\left(T_{\beta}\right)\right\} \backslash\{\lambda \in$ C ; $\left.i_{t}(\alpha, \lambda)=i_{t}(\beta, \lambda) \neq 0\right\}$, then $\alpha-\lambda=|\alpha-\lambda| z^{\ell} e^{i u}$ and $\beta-\lambda=|\beta-\lambda| z^{\ell} e^{i v}$ where $u$ and $v$ are in $C$, and $\ell$ is an integer. Hence $(\alpha-\lambda) /(\beta-\lambda)=(|\alpha-\lambda| /|\beta-\lambda|) e^{i(u-v)}$. By Theorem C, $\lambda \notin \sigma\left(S_{\alpha \beta}\right)$. This completes the proof of (4). (5) is a result of (4).

(6) If $\lambda \in \alpha(D) \backslash \mathcal{R}(\alpha) \cup \mathcal{R}(\beta)$, then $\alpha-\lambda=q h$ and $\beta-\lambda=\bar{p} \bar{k}$ where $q$ and $p$ are inner, and $h$ and $k$ are invertible in $H^{\infty}$. Hence $(\alpha-\lambda) /(\beta-\lambda)=q p h / \bar{k}$ and so by Theorem C $\lambda \in \sigma\left(S_{\alpha \beta}\right)$. This shows that $\alpha(D) \backslash \mathcal{R}(\alpha) \cup \mathcal{R}(\beta) \subseteq \sigma\left(S_{\alpha \beta}\right)$. By the same method we can show that $\beta(D) \backslash \mathcal{R}(\alpha) \cup \mathcal{R}(\beta) \subseteq \sigma\left(S_{\alpha \beta}\right)$. By (1), $[\alpha(D)]^{c l} \cup[\overline{\bar{\beta}(D)}]^{c l} \subseteq \sigma\left(S_{\alpha \beta}\right)$. If $\lambda \notin[\alpha(D)]^{c l} \cup[\bar{\beta}(D)]^{c l}$, then $\alpha-\lambda=h$ and $\beta-\lambda=\bar{k}$ where both $h$ and $k$ are invertible in $H^{\infty}$. By Theorem $\mathrm{C}, \lambda \notin \sigma\left(S_{\alpha \beta}\right)$.

(7) If $\lambda \in[\alpha(D)]^{c l} \backslash \mathcal{R}(\alpha) \cup \mathcal{R}(\beta)$, then $\alpha-\lambda=q_{\lambda} h_{\lambda}$ and $\beta-\lambda=p_{\lambda} k_{\lambda}$ where both $q_{\lambda}$ and $p_{\lambda}$ are inner and both $h_{\lambda}$ and $k_{\lambda}$ are invertible in $H^{\infty}$. Hence $(\alpha-\lambda) /(\beta-$ $\lambda)=q_{\lambda} \bar{p}_{\lambda} h_{\lambda} / k_{\lambda}$. If $T_{q_{\lambda} \bar{p}_{\lambda}}$ is not invertible, by Theorem C $\lambda \in \sigma\left(S_{\alpha \beta}\right)$. This implies that $\left\{[\alpha(D)]^{c l} \cup[\beta(D)]^{c l}\right\} \backslash\left\{\lambda \notin \mathcal{R}(\alpha) \cup \mathcal{R}(\beta) ; T_{q_{\lambda} \bar{p}_{\lambda}}\right.$ is invertible $\} \subseteq \sigma\left(S_{\alpha \beta}\right)$. If $\lambda \notin$ $[\alpha(D)]^{\text {cl }} \cup[\beta(D)]^{\text {cl }}$, then $\lambda \notin \sigma\left(S_{\alpha \beta}\right)$ as in (6). If $\lambda \notin \mathcal{R}(\alpha) \cup \mathcal{R}(\beta)$ and $\bar{T}_{q_{\lambda} \bar{p}_{\lambda}}$ is invertible, then by Theorem $\mathrm{C} \lambda \notin \sigma\left(S_{\alpha \beta}\right)$.

(8) $\sigma\left(S_{\alpha \beta}\right) \subseteq[D]^{c l}$ by $(7)$ and so if $\lambda \notin(\mathcal{R}(\alpha) \cup \mathcal{R}(\beta)) \cap[D]^{c l}$, then the inner part of $\alpha-\lambda$ is $q_{\lambda}=(\alpha-\lambda) /(1-\bar{\lambda} \alpha)$ and the inner part of $\beta-\lambda$ is $p_{\lambda}=(\beta-\lambda) /(1-\bar{\lambda} \dot{\beta})$. Then $\operatorname{sing} q_{\lambda}=\operatorname{sing} q \neq \operatorname{sing} p=\operatorname{sing} p_{\lambda}$. By [6, Theorem 1], $T_{q_{\lambda} \bar{p}_{\lambda}}$ is not invertible. By (7), this implies that $\sigma\left(S_{\alpha \beta}\right)=[\alpha(D)]^{c l} \cup[\beta(D)]^{c l}=[D]^{c l}$.

\section{References}

1. A.Devinatz, Toeplitz operators on $H^{2}$ spaces, Trans. Amer. Math. Soc., 112 (1964), 304-317.

2. R.G.Douglas, Banach Algebra Techniques In Operator Theory, Academic Press, New York, 1972. 
3. I.Feldman, N.Krupnik and I.Spitkovsky, Norms of the singular integral operator with Cauchy kernel along certain contours.

4. J.B.Garnett, Bounded analytic functions, Academic Press, New York, 1981.

5. I.Gohberg and N.Krupnik, "One-dimensional Linear Singular Integral Equations, Vol.I and Vol.II, Birkhäuser, 1992.

6. H.Helson and G.Szegö, A problem in prediction theory, Ann. Mat. Pura Appl., $51(1960), 107-138$.

7. R.Hunt, B.Muckenhoupt and R.Wheeden, Weighted norm inequalities for the conjugate function and Hilbert transform, Trans. Amer. Math. Soc., 176(1973), 227-251.

8. M.Lee and D.Sarason, The spectra of some Toeplitz operators, J. Math. Anal. Appl. 33(1971), 529-543.

9. T.Nakazi, Toeplitz operators and weighted norm inequalities, Acta Sci. Math. (Szeged), 58(1993), 443-452.

10. R.Rochberg, Toeplitz operators on weighted $H^{p}$ spaces, Indiana Univ. Math. J., 26(1977), 291-298.

11. I.Spitkovsky, Singular integral operators with PC symbols on the spaces with general weights, J.Funct. Anal. 105(1992), 129-143. 